\title{
MULTIPLE JUNCTION CELL CHARACTERIZATION USING THE LBIC METHOD: EARLY RESULTS, ISSUES, AND PATHWAYS TO IMPROVEMENT
}

\author{
Jason R. Finn, Barry R. Hansen, Jennifer E. Granata \\ Sandia National Laboratories, Albuquerque, New Mexico, USA
}

\begin{abstract}
A Light Beam Induced Current (LBIC) measurement is a non-destructive technique that produces a spatial graphical representation of current response in photovoltaic cells with respect to position when stimulated by a light beam. Generally, a laser beam is used for these measurements because the spot size can be made very small, on the order of microns, and very precise measurements can be made.
\end{abstract}

Sandia National Laboratories Photovoltaic System Evaluation Laboratory (PSEL) uses its LBIC measurement technique to characterize single junction mono-crystalline and multi-crystalline solar cells ranging from miniature to conventional sizes. Sandia has modified the already valuable $\mathrm{LBIC}$ technique to enable multi-junction $\mathrm{PV}$ cells to be characterized.

\section{INTRODUCTION}

The LBIC method is used to diagnose various manufacturing defects within a photovoltaic cell [1, 2]. Some of the issues that have been detected previously are microcracks within the substrate, problems with the metallized screen printing process, and other defects due to the manufacturing steps involved in creating a cell. These issues can cause efficiency and reliability problems at the cell level, and in turn the photovoltaic module level. It is important to note that many of the problems identified when using the LBIC method are not easily recognized by visual inspection or many of the other methods used in photovoltaic cell characterization.

Over the last two years, Sandia National Laboratories has performed numerous LBIC tests on a number of different manufacturers' cells. The technologies tested range from traditional multi-crystalline and monocrystalline silicon cells including all back contact devices; as well as other devices which have photovoltaic properties but are being used in other applications, such as Cadmium Telluride focal plane arrays used in night vision applications.

In the last several years, the interest in multi-junction cells for use in CPV applications has dramatically increased. As a result, there is a greater need for improving the diagnostics of these types of cells. The main difference between multi-junction cells and traditional silicon cells is that the cells used usually have multiple junctions instead of just one junction, as well as different materials that make up the cell. The multiple layers of materials present a complicated problem when trying to perform an LBIC scan on a multi-junction cell; specifically, how to isolate and measure each junction independently. Over the last several months Sandia has been working on the solution to this problem, and has produced some intriguing results.

\section{EXPERIMENTAL}

To perform an LBIC scan using a laser, the laser wavelength must be chosen to generate current within the material of interest. There are several lasers available to scan a typical silicon cell. Two of the more commonly used lasers are $670 \mathrm{~nm}$ and $1064 \mathrm{~nm}$, which can be used to penetrate different depths into the silicon substrate. The laser beam passes through a chopper and then through a glass convex focusing lens, and onto the cell. A chopper is used to minimize the noise in the measurement from other light sources such as the $60 \mathrm{~Hz}$ fluorescent room lighting. The focusing lens allows the spot size to be focused to approximately 8 microns in diameter. The cell is placed on a gold plated vacuum chuck, which is mounted on top of an $X-Y-Z$ axis stage. This allows precise movement of the mounted cell under the laser in the $X$ and $Y$ directions using stepper motors, while the $Z$ direction is used to focus the laser spot size.

During a measurement, the current signal is fed through a transimpedance amplifier. This converts the current signal to an $A C$ voltage signal. The $A C$ signal is then passed via a BNC cable to a lock-in amplifier. The output of the lock-in amplifier is passed via a GPIB cable to a computer. The control software for the system is a custom made LabVIEW program.

In a multi-junction (MJ) cell, the junctions are in series, which can be modeled as diodes in series. To perform an LBIC measurement on one junction, that junction must be the current limiting junction of the series. The process of performing an LBIC scan on an MJ cell is a variation of the conventional cell method. The MJ cells used in this experiment are triple junction: GalnP, GaAs, and $\mathrm{Ge}$. To isolate a specific junction for measurement, light bias is used with wavelengths in the specific regions of the other junctions, and no light bias is used in the wavelength range of the junction under test. This can be accomplished by using different combinations of optical 
high and low pass filters in the path of the light biasing source. Then, a laser is used with a wavelength that lies in the responding region of the junction of interest. As there are three junctions in the sample CPV cell assembly tested, there are three different configurations used in pinpointing these specific junctions. This setup uses two light biasing sources.

To accurately separate out the top junction, a light bias source must be able to flood the middle and bottom junctions, and a laser must be applied with a wavelength in a region of response of the first junction that will not excite the middle or bottom junctions. For testing the bottom junction, a similar method is used. The laser wavelength applied is in the response region of the bottom junction, but does not excite the top or middle junctions. Light bias is then applied using filters that allow light to flood the top and middle junctions.

Pinpointing the middle junction is more complicated. The top and bottom junctions must be flooded, and a laser wavelength must be applied that is in the region of response of the middle junction. Again, the laser wavelength must not excite the top or bottom junctions. To perform this test, there must be a short pass filter in the path of one biasing light that floods the top junction only. On the other biasing light source, there must be a long pass filter in its path that floods only the bottom junction. The area of each of the light biasing spots must be exactly overlaid so that the entire area of the cell being tested is within the light bias.

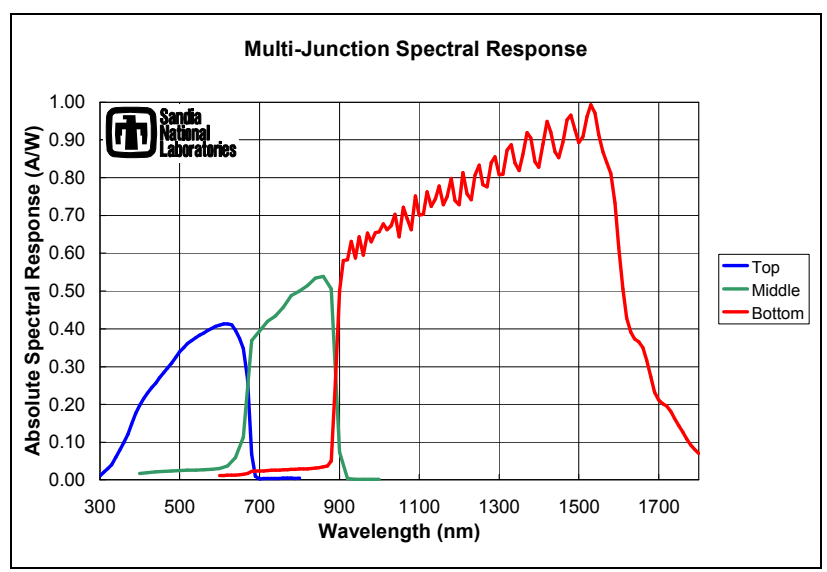

Fig. 1. A spectral response of the sample multi-junction cell assembly.

A spectral response of the sample cell was performed to help determine the exact wavelengths for the lasers and the filters to be used [2, 3]. Performing a spectral response of a multi-junction cell requires the use of the same types of low and high pass filters to separate out the junctions as for an LBIC scan. A multi-junction spectral response was performed using this method which actually consists of three different spectral response measurements, with each having different light biasing conditions. The results are shown in Figure 1.
As shown in the spectral response, the top junction response range is approximately $300 \mathrm{~nm}$ to $700 \mathrm{~nm}$; the middle junction response range is approximately $600 \mathrm{~nm}$ to $900 \mathrm{~nm}$; and the bottom junction response range is 900 $\mathrm{nm}$ to $1800 \mathrm{~nm}$. The results of this spectral response give a clear idea of what wavelengths need to be used to properly perform an LBIC scan.

During the LBIC measurement process, the top junction was scanned using the $405 \mathrm{~nm}$ laser and a 750 $\mathrm{nm}$ long pass filter for the light bias. To test the middle junction, one light source went through a $500 \mathrm{~nm}$ short pass filter, while the other light source went through an $1100 \mathrm{~nm}$ long pass filter. An $830 \mathrm{~nm}$ laser was used to excite the middle junction. For the bottom junction, an 800 $\mathrm{nm}$ short pass filter was used for the light bias and a 1310 $\mathrm{nm}$ laser was used to excite the junction.

There are several other items that make up the LBIC system. A digital laser power supply is used to accurately control the temperature and power output of each laser. For the light biasing, two 250W fiber-optic light sources and power supplies are used. Two 6-slot filter wheels are used to hold the specific filters that need to be changed during the automated testing process. A single axis motorized stage is used to mount the different lasers so the testing wavelength can be easily changed. A simplified block diagram of the LBIC equipment setup is shown in Figure 2.

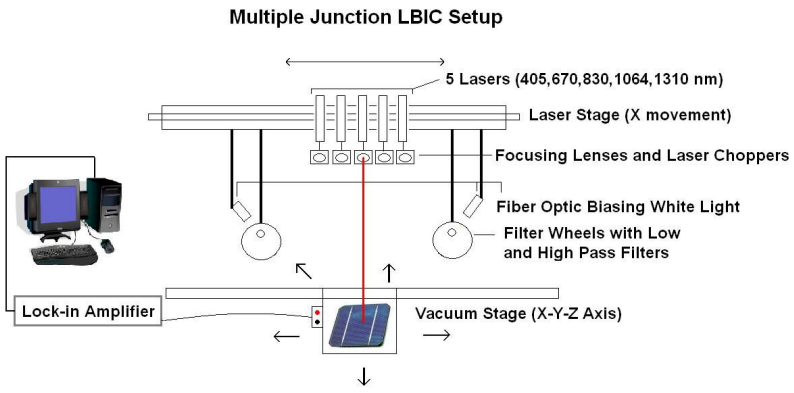

Fig. 2. Simplified block diagram of the multiple junction LBIC setup.

A typical LBIC scan begins with mounting a cell to the vacuum chuck. Once the cell is on the chuck, its dimensions are measured and input into the custom made LabVIEW program. The light bias is turned on and the filter wheel combination is adjusted for the specific junction to be tested. The laser is then turned on. Some fine tuning adjustments are made to the laser power level and the light bias intensity to assure the light bias induced current is greater than that of the laser induced current. Once the setup is complete, the cell scan is started. A typical scan for this type of cell $\left(1 \mathrm{~cm}^{2}\right)$ consists of 250000 data points at high resolution. Maximum resolution of the LBIC system is a step size of 1 micron between data points. A scan with a step size of 15 microns in both the $X$ and $Y$ directions produces a detailed scan equivalent to the maximum resolution. This is due to the laser spot size being approximately 8 microns in diameter, and a step size of less than the size of the spot will produce very similar 
results. A lesser resolution scan takes less time and is optimally taken with a step size of 25 microns. The scan is conducted starting from the top right of the scan area input in the setup process. The direction of the scan is horizontal, starting from the top to bottom rows. The scan is always conducted from right to left, and the laser returns to the right side of the scan area when a new row is started. During a specific data point measurement, the $X-Y$ stage is moved into position, and there is a $100 \mathrm{~ms}$ settling time before a reading of that location is taken. Then the data is saved to file and the $X-Y$ stage is moved again to the next location. The scan rate is approximately 250 data points per minute. Typically scans of high resolution for a cell that is $1 \mathrm{~cm}^{2}$ take approximately 14 hours to complete, whereas scans of lesser resolution take approximately 3 hours to complete.

\section{RESULTS AND DISCUSSION}

\section{LBIC Results}

LBIC scans are detailed and clearly show defects, grain boundaries (both external and internal), and problems in the metallization. Figure 3 illustrates an example of an LBIC scan taken on a typical silicon cell, which is shown in Figure 4.

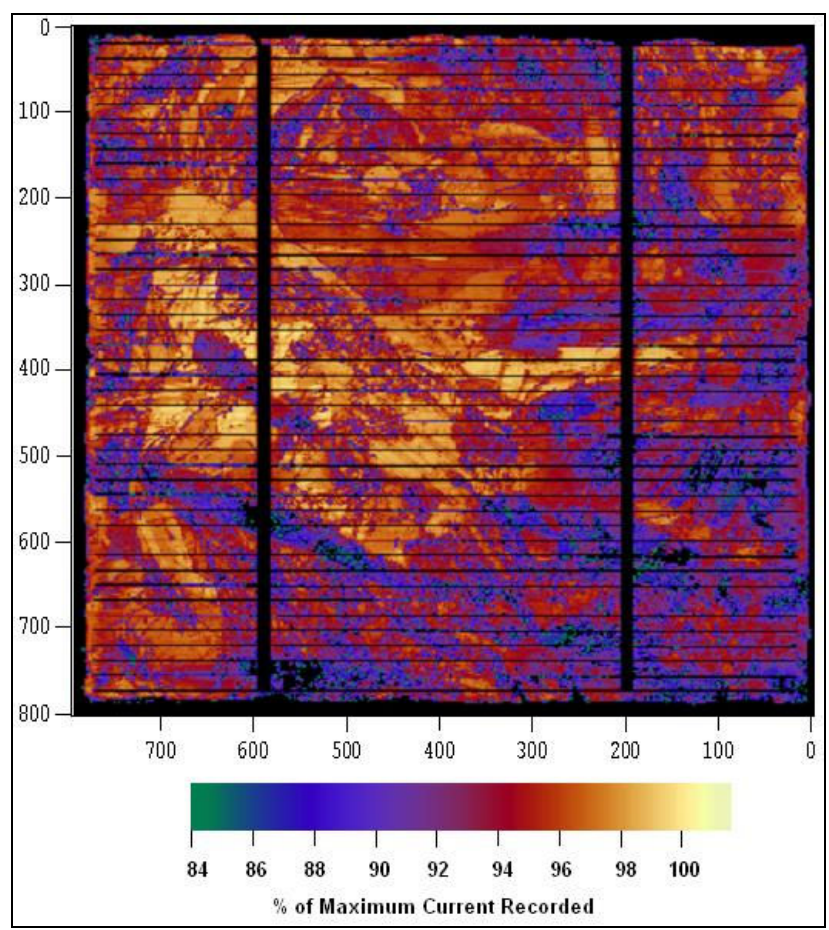

Fig. 3. LBIC performed on multi-crystalline cell.

This particular LBIC scan was taken using a $670 \mathrm{~nm}$ laser and no light bias. In the scan results, darker colors indicate less current response than lighter colors, under the identical laser beam intensity. Some of the regions of interest are the dark spots that makeup the majority of the lower right quadrant of the cell. These dark spots show up to $20 \%$ response degradation from the maximum current level recorded. Defects like the ones shown illustrate problems that affect efficiency, and are not able to be diagnosed through visual inspection techniques.

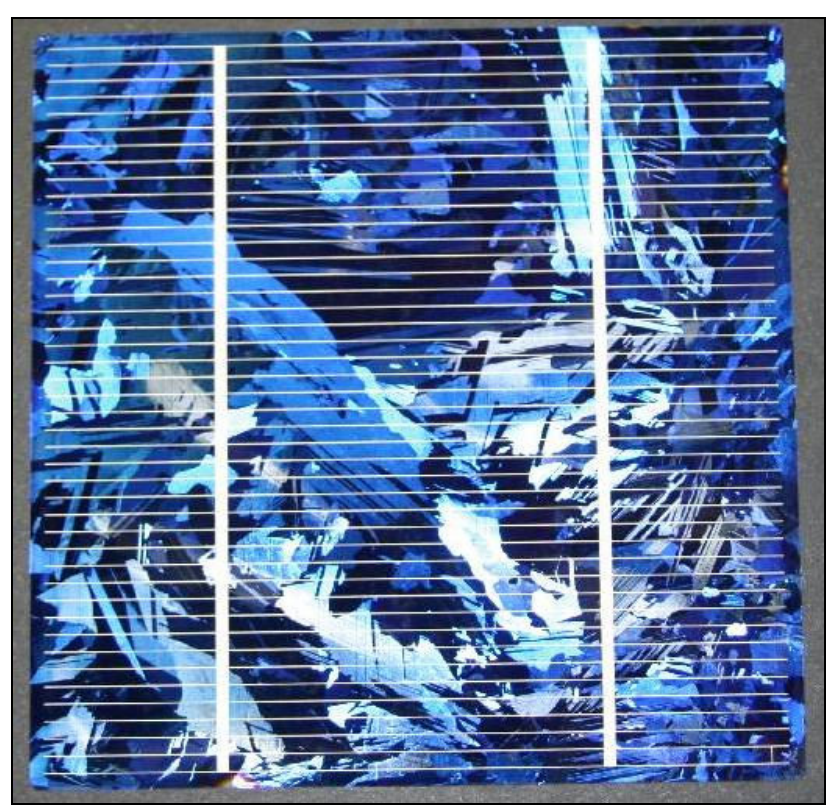

Fig. 4. Typical multi-crystalline Silicon (mc-Si) cell.

In this crystalline silicon cell, the grain boundaries can be seen for some of the features visible in Figure 4, as well as some of the deeper grain boundaries that are not visible. The metallization is clearly visible as well. In several other scans conducted on similar sample silicon cells, there have been problems detected in the screen printing process that have caused dead spots adjacent to the busbars and the gridlines.

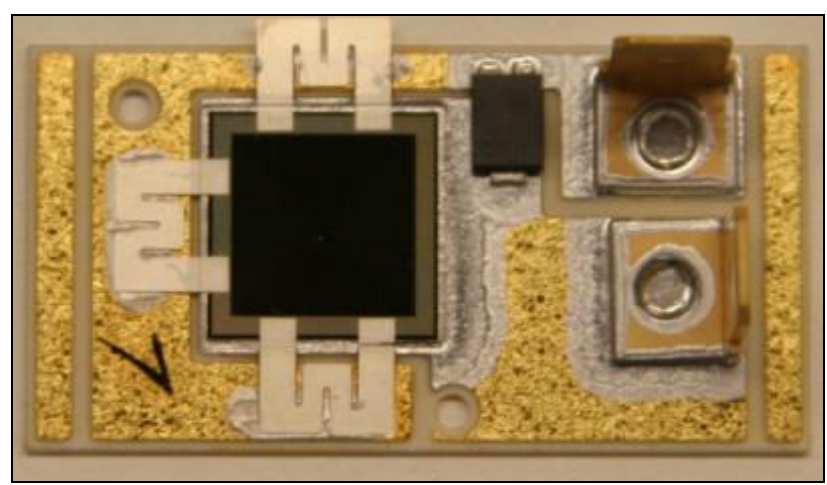

Fig. 5. Sample multi-junction cell assembly used in LBIC and spectral response testing.

To test the multi-junction LBIC scan technique, Emcore, one of Sandia's industry partners, provided a sample cell assembly, shown in Figure 5. This cell is a typical multi-junction cell that was from a lot of cells made in 2007. The technology has improved since that time, but this cell makes a good sample for the LBIC measurement.

First, a one-sun IV measurement was made on the sample cell. A one-sun IV plot is shown in Figure 6 along with the relevant IV parameters. A silicon reference cell 
was used to set up the simulator. Since the spectral response curves for silicon and the MJ cells are quite different, this introduced uncertainty in the measurement of approximately $5 \%$. This cell is well-behaved with no visible defects. In the future, cells with known defects will be tested to see what more can be diagnosed through the use of the LBIC measurement.

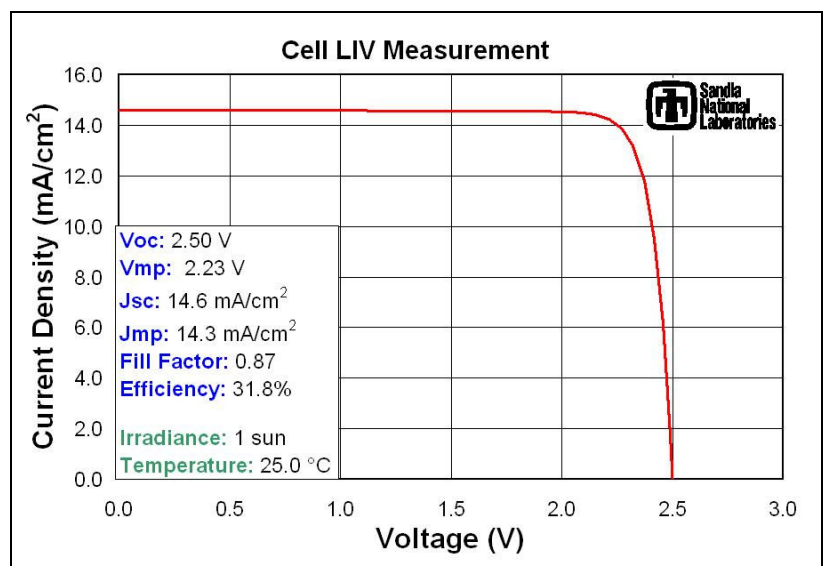

Fig. 6. Sample multi-junction cell IV measurement with additional measured and calculated parameters.

The top junction of the cell was tested first using the method described earlier in the experimental section. The results are shown in Figure 7.

In Figure 7, the darker colors indicate less of a current response, while the lighter colors indicate a stronger current response under the same laser intensity. This contrast scheme was used for all of the LBIC plots; however, the difference between the specific colors may vary in each plot. In each specific LBIC scan, the maximum current is recorded and then normalized, making the maximum current reading the "100\%" level, and no current response the " $0 \%$ " level.

One area of interest in the scan is the edge of the active area of the cell. There is a reduced current response in the edges of the cell, but only on three of the four sides. The right edge of the cell, which does not have interconnects attached to the metallization, shows a good response. This could indicate that the process used in attaching the interconnects to the sides of the cell is damaging the edges of the cell, possibly with heat or pressure when soldering. Several small defects can also be seen in this top junction at various points. Note there is a small strip of active cell area outside of the metallization region that responds to and appears on each LBIC scan.

The middle junction was tested next. The results of the middle junction scan are shown in Figure 8 . The middle junction yielded some slightly grainy results. This could be due to interference from the index of refraction difference of the materials used in the top and middle junctions. Further testing will be performed to fine tune the

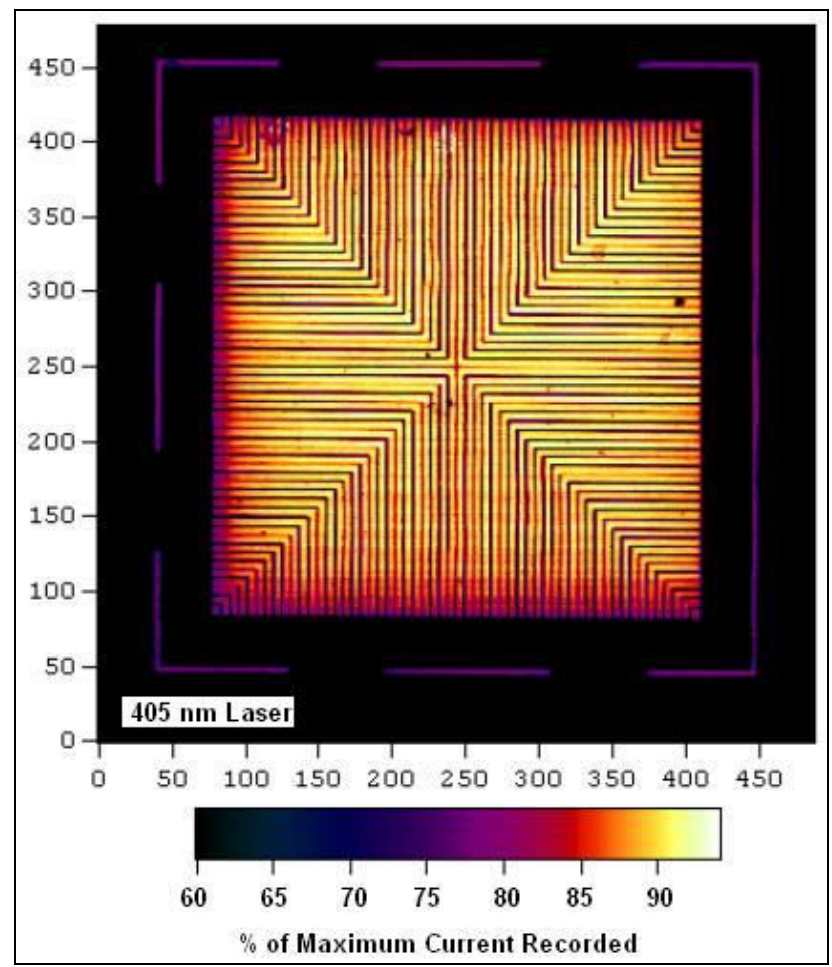

Fig. 7. Top junction LBIC scan of sample multi-junction cell assembly.

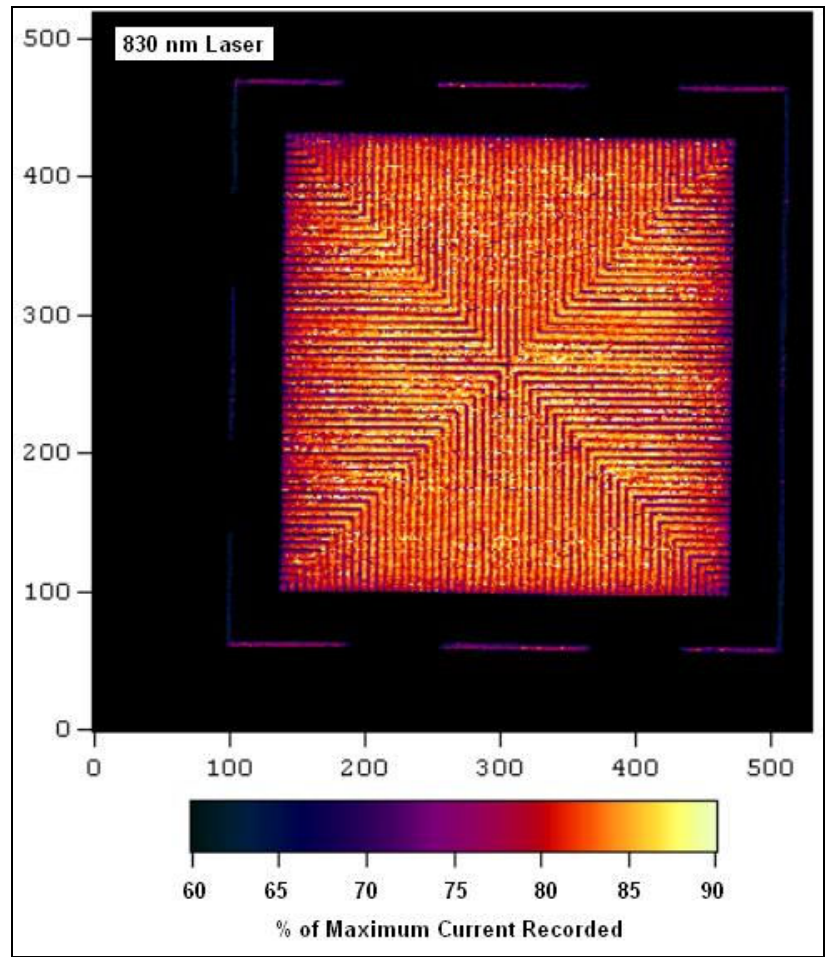

Fig. 8. Middle junction LBIC scan of sample multi-junction cell assembly. 
middle junction scan capability. In this case, all four edges have a reduced current from the maximum recorded current in this scan.

The next scan was performed on the bottom junction, shown in Figure 9. This scan yielded the most interesting results. The patterns that emerged resembled interference fringes, similar to those which appear in the spectral response taken of the sample. It was hypothesized that these patterns could be a Moiré pattern [4], which is interference when a line crosses with another line in a scan at a slight angle. However, the results were repeatable, even when using different step sizes and scan angles. A scan of another similar cell's bottom junction yielded a completely different but repeatable pattern. If the gridlines were the source of the interference, then it would seem that the pattern would be symmetrical at the four corners of the sample, which was not the case. Another interesting detail is that the lines appear to be extending under the metallization and out to the small section of active area at the edge of the cell. This would be another indication that the gridlines are not the source of the interference.

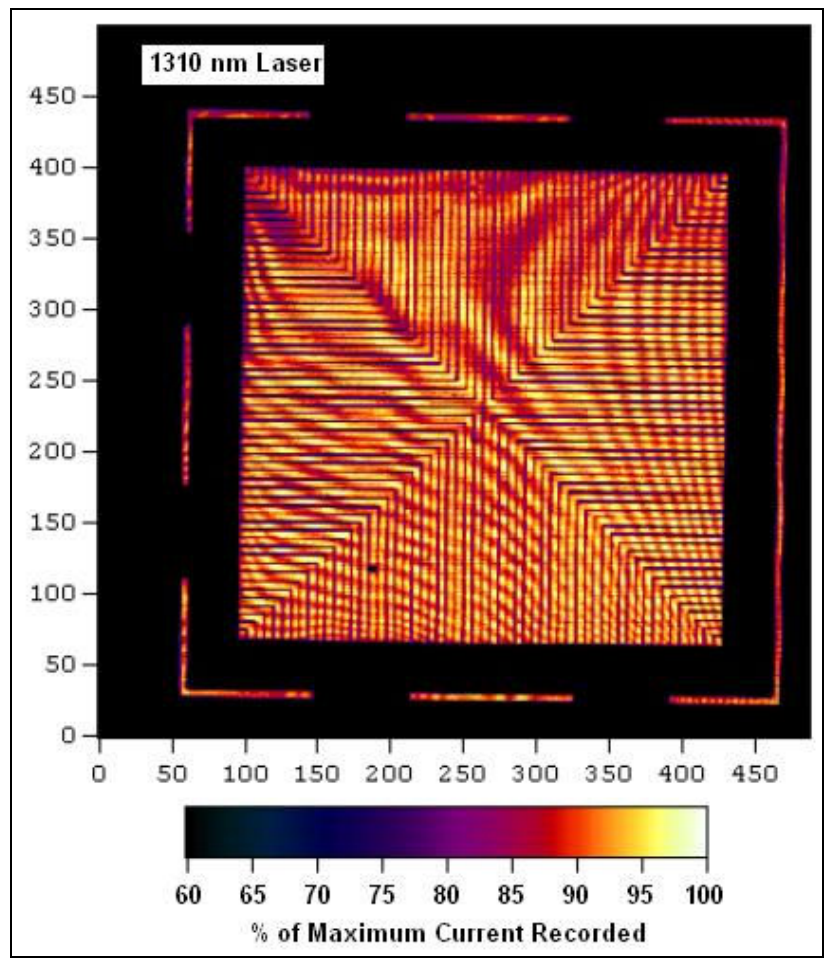

Fig. 9. Bottom junction LBIC scan of sample multi-junction cell assembly.

The previous scans were taken with a 15 micron step size, and the cell edge nearly squared to the incident beam. For the purposes of this paper, this will be considered 0 degrees. To test the repeatability of the results for the bottom junction, the cell orientation was changed to 30 degrees off from the previous measurements, and the step size was changed to 20 micron increments. The results are shown in Figure 10.
Although there is some interference visible in the scan that does resemble the Moiré pattern around the gridlines, the pattern underneath remains exactly as before. This suggests that the pattern is being created by something that lies within the cell and is not an optical artifact from the measurement technique. It is possible this pattern is caused by interference between the thin layers of the top and middle junctions. More testing will be done in the future to determine exactly what is causing these types of patterns. In addition, tests will be conducted on other types of multi-junction cells to see if similar patterns emerge.

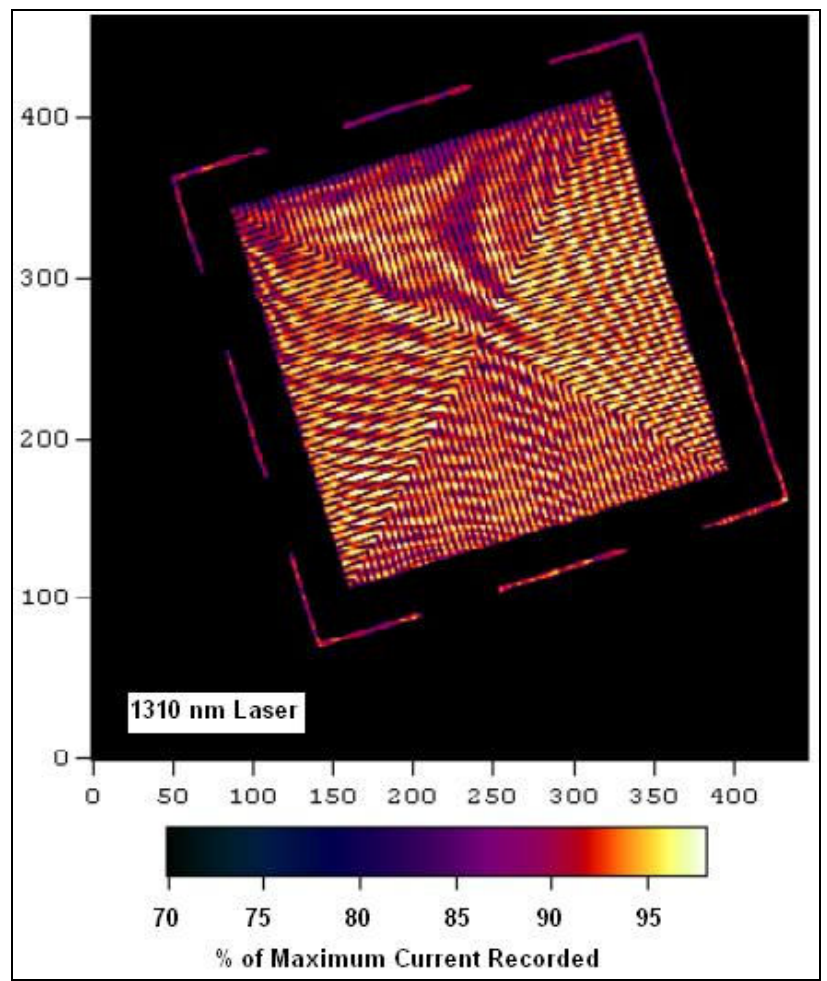

Fig. 10. Bottom junction LBIC scan of sample cell tilted 30 degrees, and with a 20 micron step size.

\section{Spectral Response}

The spectral response measurement is useful for determining the correct lasers and filters to use for the LBIC scan. It is also important in determining the current balance within a multi-junction solar cell. The quantum efficiency and current density for each junction were calculated from the spectral response. The results are shown in Figure 11.

The calculated current densities for each junction are: $\mathrm{JsC}_{\text {Top }}=14.12 \mathrm{~mA} / \mathrm{cm}^{2}$; $\mathrm{Jsc}_{\text {Middle }}=12.24 \mathrm{~mA} / \mathrm{cm}^{2}$; and $\mathrm{JsC}_{\text {Bottom }}=19.70 \mathrm{~mA} / \mathrm{cm}^{2}$. This would indicate the middle cell as the current-limiter. The short-circuit current measured under one-sun conditions is $14.62 \mathrm{~mA} / \mathrm{cm}^{2}$. This confirms that the LIV measurement is inaccurate due to using a silicon cell to calibrate the spectrum, suggesting the spectrum used is red-rich. The QE curves also demonstrate likely current leakage during both the spectral 
response and LBIC measurements, providing insight into areas for improving the measurement technique.

The PSEL is the Spectral Response Primary Calibration site at Sandia National Laboratories. Using a primary reference for spectral response allows for an absolute spectral response measurement, not just a relative measurement. This means that the short-circuit current calculated from quantum efficiency curves is also absolute, to within error of the spectrum used for calculation. Such a measurement and analysis technique can be of great use in determining the short-circuit current of multi-junction solar cells and quantifying the Jsc ratios among the cells in the stack. This is particularly relevant for MJ cells when the solar simulators used for LIV testing are not well-matched to the solar spectrum.

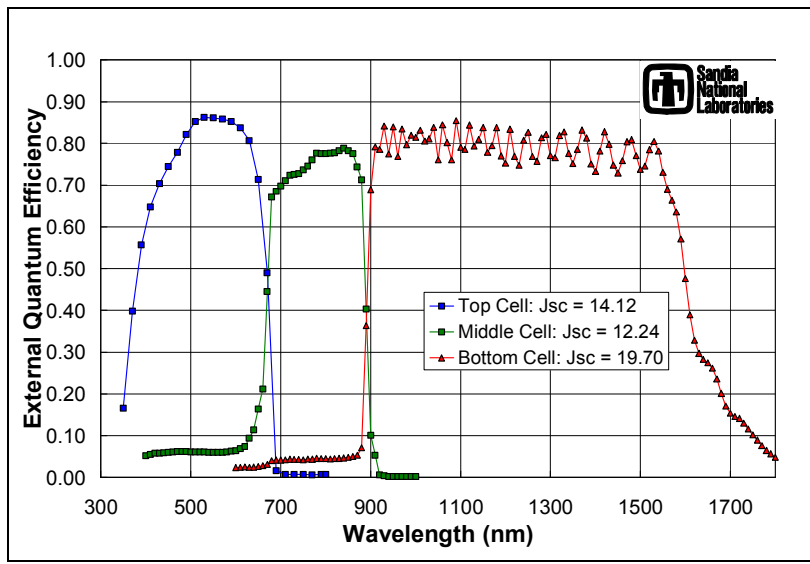

Fig. 11. Calculated quantum efficiency and short-circuit current for each junction for the multi-junction cell assembly.

\section{CONCLUSIONS}

Sandia National Laboratories has successfully demonstrated the ability to accurately characterize each junction in a multi-junction solar cell using the LBIC technique. This technique allows multi-junction solar cell designers and manufacturers to perform in-depth diagnostics on each junction. This technique can diagnose non-uniform cell response, microcracking, metallization defects, and other material or growth-driven imperfections which reduce power output that are not easily identified using existing diagnostics. In addition, the spectral response measurement allows the specific junctions in a multi-junction cell to be characterized as an absolute measurement. These two testing capabilities will allow multi-junction cell manufacturers to optimize their manufacturing process and produce cells with greater efficiencies as a result.

\section{REFERENCES}

[1] Jan Salinger, Vitezslav Benda, and Zdenek Machacek, "A Note on Solar Cell Diagnostics Using LBIC and LBIV Methods", Proc. $26^{\text {th }}$ International Conf. on Microelectronics, 2008
[2] D. L. King, B. R. Hansen, J. M. Moore, D. J. Aiken, "New Methods for Measuring Performance of Monolithic Multi-Junction Solar Cells", Proc. 28 ${ }^{\text {th }}$ IEEE Photovoltaic Spec. Conf., 2000, pp. $1197-1201$

[3] M. Pravettoni, A. Virtuani, H. Müllejans, R. P. Kenny, E. D. Dunlop, K. W. J. Barnham, "Spectral Response Measurements of Multi-Junction PV Modules at the European Solar Test Installation Laboratories and Their Usage in Clear Day Outdoor Characterisation", $23^{\text {rd }}$ European Photovoltaic Solar Energy Conference, 2008, pp. 2908 - 2912

[4] Emin Gabrielyan, "The Basics of Line Moiré Patterns and Optical Speedup", Retrieved May 22, 2009, from: http://switzernet.com/people/emin-gabrielyan/070306optical-speedup

\section{ACKNOWLEDGEMENTS}

Sandia is a multiprogram laboratory operated by Sandia Corporation, a Lockheed Martin Company, for the United States Department of Energy's National Nuclear Security Administration under contract DE-AC0494AL85000. Sandia acknowledges the support of the DOE Solar Energy Technologies Program in particular for the work presented here.

Sandia would like to thank Emcore for supplying the sample multi-junction cells used in the development of this testing capability. 